\title{
Resistance of Helicobacter pylori to tetracycline, amoxicillin, clarithromycin and metronidazole in Israeli children and adults
}

\author{
Avi Peretz ${ }^{1}$, Maya Paritsky ${ }^{2}$, Omar Nasser ${ }^{1}$, Diana Brodsky ${ }^{1}$, Tatyana Glyatman ${ }^{1}$, Sofia Segal ${ }^{3}$ and Avi On ${ }^{3}$
}

The aim of this study was to examine Helicobacter pylori-resistance rate to different antibiotics: tetracycline, amoxicillin, clarithromycin and metronidazole, and to compare eradication rates in adults and children in Israel. The study was based on the hypothesis of high-resistance rates to clarithromycin and metronidazole especially in adults and overall low-resistance rates to tetracycline and amoxicillin. One seventy six biopsies from patients with dyspeptic symptoms were cultured of which 100 were from adults (19-79 years) and 76 from children (7-17 years). All positive cultures were examined by Epsilometer test for MIC determination against tetracycline, amoxicillin, clarithromycin and metronidazole. $48.3 \%$ (85 out of 176 ) were $H$. pylori positive, of which $44 \%$ were from adults and $54 \%$ from children. Antibiotic resistance was seen in 31 out of $44(70.5 \%)$ for metronidazole, 1 out of $44(2.3 \%)$ for amoxicillin, 10 out of $44(22.3 \%)$ for clarithromycin and 1 out of $44(2.3 \%)$ for tetracycline among adults. Antibiotic resistance was seen in 10 out of $41(24.4 \%)$ for metronidazole, 5 out of $41(12.2 \%)$ for amoxicillin, 10 out of $41(24.4 \%)$ for clarithromycin and 1 out of $41(2.4 \%)$ for tetracycline among children. High rates of $H$. pylori resistance to metronidazole and clarithromycin was found especially among adults. Therefore, to increase the success rate of anti- $\boldsymbol{H}$. pylori treatment, other classes of antibiotics need to be considered.

The Journal of Antibiotics (2014) 67, 555-557; doi:10.1038/ja.2014.38; published online 30 April 2014

\section{INTRODUCTION}

Helicobacter pylori (H. pylori) infection is one of the most common bacterial infections of the digestive system. H. pylori infection is an important factor in the development of serious upper gastrointestinal diseases: gastritis, duodenitis, gastric ulcers, duodenal ulcers (reported to develop in $1-10 \%$ of infected patients), gastric cancer (in $0.1-3 \%$ ) and gastric mucosa-associated lymphoid-tissue lymphoma (in $<0.01 \%) .{ }^{1,2}$ Unfortunately, very little is known about the details of the modes of transmission of $H$. pylori and its routes of dissemination. The primary modes of transmission are thought to be fecal-oral and oral-oral, but some indirect evidence reports transmission via drinking water and other environmental sources. ${ }^{3,4}$ The current most effective treatment includes proton-pump inhibitors in combination with two antibiotics. However, owing to bacterial resistance to antibiotic therapy, $\sim 20 \%$ of patients fail to respond to the eradicative first-line treatment and remain $H$. pylori positive. ${ }^{5}$ Prevalence of resistance to treatment is variable in different areas of the world and probably is related to indication, particularly for drugs that are used in the treatment of respiratory tract infections. For instance, in countries where the use of clarithromycin is rare, the resistance rate is low, while in countries where its use is wide, the resistance rate reaches $10-15 \% .{ }^{6,7}$ Similarly, metronidazole-resistance rate is much higher in developing countries (50-80\%), for example in Mexico it is $76.3 \%$, which is in contrast to a much lower-resistance rate in Japan (9-12\%). ${ }^{8}$ Additionally, resistance rates between adults and children are different. ${ }^{7}$

The aim of this study was to examine $H$. pylori-resistance rate in response to different antibiotics: tetracycline, amoxicillin, clarithromycin and metronidazole, and to compare response rate in adults and children in Israel. The study was based on the hypothesis of high-resistance rates to clarithromycin and metronidazole especially in adults and overall low-resistance rates to tetracycline and amoxicillin, reflected in treatment success or failure.

\section{MATERIALS AND METHODS}

\section{Bacterial culture}

The study population consisted of 176 patients with dyspeptic symptoms. Among them 71 were males and 105 females of which 100 were adults (19-79 years) and 76 children (7-17 years). All patients did not receive antibiotics in the months preceding the study. Gastric biopsies from the antrum and the corpus were taken from each patient. All samples were collected in the period June 2011-April 2012. The biopsies were cultured within $60 \mathrm{~min}$ on $\mathrm{H}$. pyloriselective medium (Hy-lab, Rehovot, Israel) enriched with 5\% horse blood/ laked-lysed supernatant and contained vancomycin, trimethoprim and

${ }^{1}$ Clinical Microbiology Laboratory, Baruch Padeh Medical Center, Poria, Affiliated to the Faculty of Medicine, Bar Ilan University, Tiberias, Israel; ${ }^{2}$ Gastrointestinal Unit, Baruch Padeh Medical Center, Poria, Affiliated to the Faculty of Medicine, Bar Ilan University, Tiberias, Israel and ${ }^{3}$ Pediatric Gastrointestinal Unit, Baruch Padeh Medical Center, Poria, Affiliated to the Faculty of Medicine, Bar Ilan University, Tiberias, Israel

Correspondence: Dr A Peretz, Clinical Microbiology Laboratory, Baruch Padeh Medical Center, Poria, Affiliated to the Faculty of Medicine, Bar Ilan University, Lower galil, Tiberias 14324, Israel. 


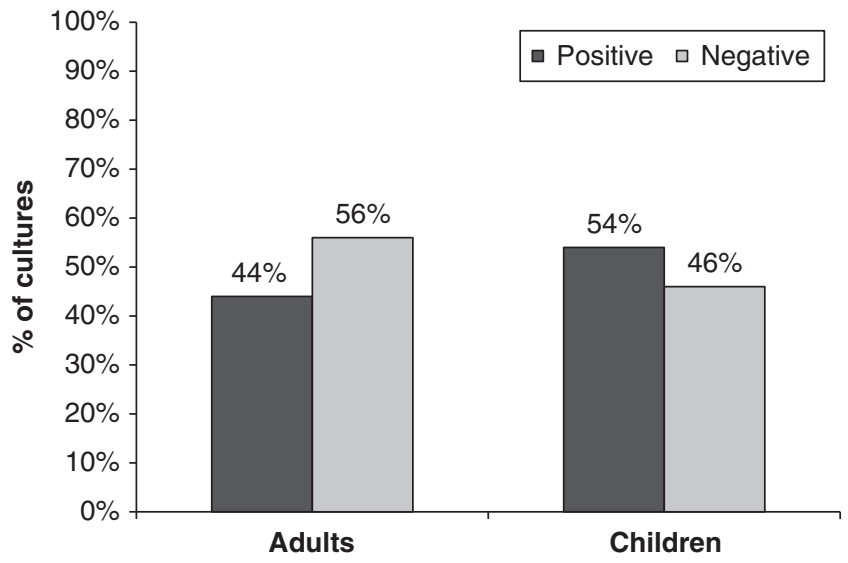

Figure 1 Helicobacter pylori prevalence among children and adults.

polymixin B to inhibit the growth of other microorganisms. Cultures were incubated for up to 8 days at $37^{\circ} \mathrm{C}$ in microaerobic atmosphere $\left(5 \% \mathrm{O}_{2}\right.$ and $\left.10 \% \mathrm{CO}_{2}\right) . \mathrm{H}$. pylori identification was carried out using Gram stain, positive oxidase, catalase and urease tests.

\section{Determination of in vitro susceptibility}

Suspensions from primary plates were prepared in $0.85 \% \mathrm{NaCl}$ solution to a 2.0 McFarland standard (containing $1 \times 10^{7}$ to $1 \times 10^{8} \mathrm{CFU}$ per $\mathrm{ml}$ ) to perform the E-test on Mueller-Hinton agar with 5\% sheep blood (Hy-lab) against tetracycline, amoxicillin, clarithromycin and metronidazole, and were incubated for $72 \mathrm{~h}$ at $37^{\circ} \mathrm{C}$ in microaerobic atmosphere. An isolate was considered resistant to amoxicillin if MIC was $>1 \mathrm{mgl}^{-1}$, to clarithromycin if MIC was $\geqslant 1 \mathrm{mgl}^{-1}$, to metronidazole if MIC was $>4 \mathrm{mgl}^{-1}$ and to tetracycline if MIC was $>2 \mathrm{mgl}^{-1} .9,10$ For MIC quality control the $H$. pylori 43504 strain was used.

\section{Statistical analysis}

For comparison between the subgroups, the analysis of variance single factor anal, $\chi^{2}$-test, Fisher's exact test and $t$-test were used appropriately. A $P$-value of $<0.05$ was considered significant.

\section{RESULTS}

Among 176 samples, 85 (48.3\%) were $H$. pylori positive of which 44 (44\%) were from adults (average age 48 years) and 41 (54\%) from children (average age 12 years) (Figure 1). All patients' positive cultures were accepted both from the antrum biopsy and from corpus biopsy. Antibiotic resistance was seen in 31 out of $44(70.5 \%)$ for metronidazole (mean MIC $8 \mu \mathrm{g}), 1$ out of $44(2.3 \%)$ for amoxicillin (MIC $3 \mu \mathrm{g}$ ), 10 out of $44(22.3 \%)$ for clarithromycin (mean MIC $4 \mu \mathrm{g})$ and 1 out of $44(2.3 \%)$ for tetracycline (MIC $4 \mu \mathrm{g}$ ) in adults. Combined resistance was observed in one sample for tetracycline and metronidazole and in nine samples for metronidazole and clarithromycin among adults. Antibiotic resistance was observed in 10 out of $41(24.4 \%)$ samples for metronidazole (mean MIC $3 \mu \mathrm{g}$ ), 5 out of $41(12.2 \%)$ for amoxicillin (mean MIC $4 \mu \mathrm{g}$ ), 10 out of 41 (24.4\%) for clarithromycin (mean MIC $3 \mu \mathrm{g}$ ) and 1 out of $41(2.4 \%)$ for tetracycline (MIC $6 \mu \mathrm{g}$ ) among children. Combined resistance was observed in one isolate for amoxicillin and metronidazole and in three samples in metronidazole and clarithromycin in children (Tables 1 and 2) ( $P$-value of 0.037 was significant). Patients who were diagnosed as positive for $H$. pylori, received triple (two antibiotics and a proton-pump inhibitor) therapy according to the results of the sensitivity tests. In patients who were resistant to two
Table 1 Pattern of primary-bacterial resistance toward different antibiotics in all of the subjects $(n=85)$

\begin{tabular}{lc}
\hline Antibiotic & Resistance, $\mathrm{n}(\%)$ \\
\hline Metronidazole & $41(48.8)$ \\
Clarithromycin & $20(23.5)$ \\
Amoxicillin & $6(7)$ \\
Tetracycline & $2(2.3)$ \\
Metronidazole \& clarithromycin & $12(14.1)$ \\
Metronidazole \& tetracycline & $1(1.2)$ \\
Metronidazole \& amoxicillin & $1(1.2)$ \\
\hline
\end{tabular}

Table 2 Pattern of primary-bacterial resistance toward different antibiotics divided into age groups

\begin{tabular}{lcc}
\hline Antibiotic & $\begin{array}{c}\text { Adults, } \mathrm{n}=44 \\
\text { Resistance (\%) }\end{array}$ & $\begin{array}{c}\text { Children, } \mathrm{n}=41 \\
\text { Resistance (\%) }\end{array}$ \\
\hline Metronidazole & $31(70.5)$ & $10(24.3)$ \\
Clarithromycin & $10(22.3)$ & $10(24.3)$ \\
Amoxicillin & $1(2.3)$ & $5(12.2)$ \\
Tetracycline & $1(2.3)$ & $1(2.4)$ \\
Metronidazole \& clarithromycin & $9(20.4)$ & $3(7.3)$ \\
Metronidazole \& tetracycline & $1(2.3)$ & $1(2.4)$ \\
Metronidazole \& amoxicillin & $0(0)$ & $0(0)$ \\
\hline
\end{tabular}

of the three antibiotics from the first-line treatment, levofloxacin was used as a part of combined therapy.

\section{DISCUSSION}

H. pylori infection is very common, and it is one of the most frequent bacterial infections of the digestive system in the world. ${ }^{1,2}$ Usually, the disease has chronic features unless it is treated by the combination of two proper antibiotics with proton-pump inhibitor or $\mathrm{H}_{2}$ receptor antagonist. $H$. pylori management in the clinical practice remains a challenge for the physicians. Choice of appropriate antibiotics is crucial in the success of treatment and recovery from $H$. pylori-related diseases. The available tests for the diagnosis of $H$. pylori infection are generally divided into invasive methods (based on gastric specimens for histology, culture or other methods) and noninvasive methods (based on peripheral samples, such as blood, breath samples, stools, urine or saliva for detection of antibodies, bacterial antigens or urease activity). Usually, the treatment prescription is empirical. Bacterial culture and antibiotics sensitivity are not a part of routine checkup, but it is recommended in case of treatment failures. ${ }^{11,12}$ The first-line treatment includes proton-pump inhibitor, and two of the three antibiotics: amoxicillin, clarithromycin or metronidazole. In cases of first-line treatment failure, either a bismuth-containing quadruple therapy or levofloxacin-containing triple therapy is recommended as second line treatment. ${ }^{13,14}$ Knowledge of the sensitivity of $H$. pylori in specific geographic areas and among specific age group can significantly improve the treatment success rates. In the present study, we observed significantly high rate of $H$. pylori resistance to metronidazole and clarithromycin. Significantly higher rate of metronidazole resistance was seen among adults: however, resistance to clarithromycin was comparable in both adults and children. On the other hand, isolates from children showed high (but nonsignificant) resistance rate to amoxicillin when compared with adults. The rate of resistance to tetracycline in both groups was significantly low. 
The resistance rates for each antibiotic may result from the before widespread use of antibiotics used in the treatment of other infections. A similar study that was conducted in Israel in 1999 showed highresistance rate to metronidazole (67\%), comparable with the rates established in the present study. Contrary to our findings, clarithromycin-resistance rate was relatively low (10\%) and amoxicillin-resistance rate was not observed. In summary, amoxicillin- and clarithromycin-resistance rates increased since 1999 while metronidazole-resistance rate remained high and unchanged in Israel. ${ }^{15}$ The low-resistance rate of $H$. pylori to tetracycline in Israel is similar to that observed in many other countries throughout the world. ${ }^{16}$ Unfortunately, use of tetracyclines is limited in pediatric patients owing to their side effects, and tetracycline-based regimens usually require the concomitant use of preparations containing bismuth salts, which are no longer available in a number of European countries owing to possible side effects. ${ }^{17}$ In conclusion, to raise the success rate of anti-H. pylori treatment, $H$. pylori cultures should be tested for antibiotic sensitivity. Second line of treatment antibiotics are currently used to treat patients with resistant $H$. pylori. Additionally we suggest replicating the study using a larger sample size to acquire information concerning $H$. pylori-resistance rates in Israel.

\section{CONFLICT OF INTEREST}

The authors declare no conflict of interest.

1 Kenneth, E. L. McColl. Clinical practice: Helicobacter pylori infection. New Engl. J. Med. 362, 1597-1604 (2010).
2 Blaser, M. J. \& Atherton, J. C. Helicobacter pylori persistence: biology and disease. J. Clin. Invest. 113, 321-333 (2004).

3 Magalhaes Queiroz, D. M. \& Luzza, F. Epidemiology of Helicobacter pylori infection. Helicobacter 11(suppl 1), 11-15 (2006).

4 Delport, W. \& van der Merwe, S. W. The transmission of Helicobacter pylori: the effects of analysis method and study population on inference. Best Pract. Res. Clin. Gastroenterol. 21, 215-236 (2007).

5 Gisbert, J. P. \& Pajares, J. M. Review article: Helicobacter pylori 'rescue' regimen when proton pump inhibitor-based triple therapies fail. Aliment Pharm. Ther. 16, 1047-1057 (2002).

6 DeKaster, E. et al. Helicobacter pylori resistance to macrolides increases, to imidazole remains stable. Gastroenterol. 112, A99 (1997).

7 Crone, J. et al. Helicobacter pylori in children and adolescents: Increase of primary clarithromycin resistance, 1997-2000. J. Pediatr. Gastroenterol. Nutr. 36, 368-371 (2003).

8 Megraud, F. H. pylori antibiotic resistance: prevalence, importance and advance in testing. Gut 53, 1374-1384 (2004).

$9 \mathrm{CISI}$. Method for antimicrobial dilution and disk susceptibility testing of infrequently isolated or fastidious bacteria; approved guideline- second edition. M45-A2:30 No.18 (Clinical and Laboratory Standards Institute, Pennsylvania, USA, 2012).

10 BSAC. Methods for antimicrobial susceptibility testing. version 7 (2008)

11 Holmes, K. P., Fang, J. C. \& Jackson, B. R. Cost-effectiveness of six strategies for Helicobacter pylori diagnosis and management in uninvestigated dyspepsia assuming a high resource intensity practice pattern. BMC. Health Serv. Res. 10, 334-340 (2010).

12 McNulty, C. A. M., Lehours, P. \& Mé graud, F. Diagnosis of Helicobacter pylori Infection. Helicobacter 16(suppl. 1), 10-18 (2011).

13 O'Connor, A. et al. Treatment of Helicobacter pylori Infection. Helicobacter 15, 46-52 (2010).

14 Malfertheiner, P. et al. Management of Helicobacter pylori infection- the Maastricht IV/ Florence consensus report. Gut. 61, 646-664 (2012).

15 Avidan, B. et al. Sensitivity and resistance of Helicobacter pylori to antibiotic treatment. Harefuah 137, 272-274 (1999).

16 De Francesco, V. et al. Worldwide $H$. pylori antibiotic resistance: a systematic review. J. Gastrointestin. Liver. Dis. 19, 409-414 (2010).

17 Gisbert, J. P. et al. H. pylori Study Group of the Asociación Española de Gastroenterología. Second-line rescue therapy with levofloxacin after H. pylori treatment failure: a Spanish multicenter study of 300 patients. Am. J. Gastroenterol. 103, 71-76 (2008). 